\title{
Editorial: Structure, Function and Evolution of Complex Cellular Organization in Bacteria and Archaea
}

\author{
Josef D. Franke ${ }^{1}$, John A. Fuerst ${ }^{2}$ and Anthony M. Poole ${ }^{3 *}$ \\ ${ }^{1}$ Department of Biology, Creighton University, Omaha, NE, United States, ${ }^{2}$ School of Chemistry and Molecular Biosciences, \\ The University of Queensland, St. Lucia, QLD, Australia, ${ }^{3}$ School of Biological Sciences, University of Auckland, Auckland, \\ New Zealand
}

Keywords: compartmentalization, organelle, nucleus, planctomycete, jumbophage, endospore, phagocytosis, autogenous

\section{Editorial on the Research Topic}

\section{Structure, Function and Evolution of Complex Cellular Organization in Bacteria and Archaea}

OPEN ACCESS

Edited by:

Haike Antelmann,

Freie Universität Berlin, Germany

Reviewed by:

Fabian M. Commichau,

Brandenburg University of Technology Cottbus-Senftenberg, Germany

*Correspondence:

Anthony M. Poole

a.poole@auckland.ac.nz

Specialty section:

This article was submitted to Microbial Physiology and Metabolism,

a section of the journa

Frontiers in Microbiology

Received: 01 August 2021 Accepted: 10 August 2021

Published: 30 August 2021

Citation:

Franke JD, Fuerst JA and Poole AM (2021) Editorial: Structure, Function

and Evolution of Complex Cellular

Organization in Bacteria and Archaea.

Front. Microbiol. 12:751416.

doi: 10.3389/fmicb.2021.751416
New discoveries are transforming our understanding of cell structure and function in Bacteria and Archaea. Whereas previously it has been assumed that complex cell ultrastructure was the exclusive domain of eukaryotes, it is becoming clear that all three domains bear organellar structures, complex intracellular membranes (Grant et al., 2018), and can produce extracellular vesicles (Gill et al., 2019). A recent survey of bacterial cell ultrastructure (Dobro et al., 2017) revealed many diverse, uncharacterized cellular features and structures, suggesting that there is still much to discover. The papers in this Research Topic highlight some of the exciting new developments in our understanding of bacterial and archaeal cell organization, evolution, and architecture.

A central question is whether complex cell architectures in Bacteria and Archaea shed light on the evolutionary origins of eukaryotic cell architecture. On the one hand, mounting evidence for cellular complexity in the newly-discovered Asgard lineages (Zaremba-Niedzwiedzka et al., 2017; Imachi et al., 2020) may help bridge the gap between archaea and the origin of the eukaryotic endomembrane system. However, it is less clear how or whether bacterial ultrastructure relates to eukaryote cellular complexity. One fascinating possibility is that some structures have evolved independently, perhaps converging on similar solutions from very different starting points (Hendrickson and Poole, 2018). A clear example of this is highlighted by the formation of a nucleus-like barrier during jumbophage infection of Pseudomonas. This is the subject of a review by Chaikeeratisak et al. The discovery of a "phage nucleus" is exciting in that it indicates there may be multiple circumstances where genetic material is compartmentalized from other parts of a cell. A stunning feature of this structure is that, in contrast to the eukaryote nucleus, this barrier is proteinaceous. The initial, mid cell positioning of the phage nucleus, and its later rotation during new phage assembly, is mediated by a spindle composed of phage-encoded proteins including PhuZ, which is evolutionarily related to tubulin.

Proteinaceous compartments are likely to be a general feature of bacteria, as reviewed by Asija et al., who examine the distribution of bacterial microcompartments (BMCs) based on a survey of the human microbiome. In contrast to jumbophage, which create a shell that sequesters the phage genome to prevent degradation by host-encoded defense mechanisms, some BMCs serve to sequester toxic aldehyde intermediates formed during metabolic reactions that occur within these compartments.

Sequestration of metabolic reactions is also a feature of membrane-bounded bacterial compartments, including anammoxosomes from planctomycetes (Neumann et al., 2014). The 
phylum Planctomycetes is diverse with a range of complex membrane architectures (Fuerst and Sagulenko, 2011; Wiegand et al., 2020), and Seeger et al. add to this catalog. They present a detailed study on Tuwongella immobilis, a relative of Gemmata obscuriglobus. Gemmata has been the subject of extensive study regarding its complex cell ultrastructure, including debate as to whether it contains a membrane-bounded genetic compartment (Santarella-Mellwig et al., 2013; Sagulenko et al., 2014, 2017; Wiegand et al., 2018). The present work, which made use of FIB-SEM (Focused Ion Beam-Scanning Electron Microscopy) tomography, suggests that T. immobilis does not contain such a compartment. Rather, Seeger et al. paint a picture of a complex intracellular membrane, replete with tunnels and caves, which they speculate may create environments where different molecular processes may be spatially separated. The question of whether any bacteria possess nucleus-like compartmentation remains an active area of investigation, with a recent isolate of the candidate phylum Atribacteria containing structures (Katayama et al., 2020) perhaps consistent with genetic compartmentation (Katayama et al., 2020; van Teeseling and Jogler, 2021). In considering the challenges of identifying bacterial genetic compartments, it is helpful to recall that the eukaryote nucleus is a dynamic structure, being disassembled in many species during mitosis (Güttinger et al., 2009). Thus, it will be interesting to understand not only whether there is genetic compartmentation in bacteria, but also whether this is stable or dynamic.

Another underappreciated facet of prokaryote cell biology is the formation of intercellular bridges, enabling cellcell communication and gene transfer. New work from Sivabalasarma et al. reveals the extent to which this occurs. Using a combination of electron cryotomography (cryoEM) and fluorescent microscopy, they report that the archaeon Haloferax volcanii transports a range of macromolecular complexes including ribosomes across these bridges, connecting the cytoplasm of mating cells.

Beskrovnaya et al. review and compare endospore formation in firmicutes with actinobacterial exospore formation. Their work suggests that endospore formation in firmicutes is ancestral, whereas exospore formation likely evolved following actinobacterial diversification.

Endospore formation is noteworthy within the context of compartmentalization for two reasons. First, endospore

\section{REFERENCES}

Angert, E. R., and Clements, K. D. (2004). Initiation of intracellular offspring in Epulopiscium. Mol. Microbiol. 51, 827-835. doi: 10.1046/j.1365-2958.2003.03869.x

Devos, D. P. (2021). Reconciling asgardarchaeota phylogenetic proximity to eukaryotes and planctomycetes cellular features in the evolution of life. Mol. Biol. Evol. 28:msab186. doi: 10.1093/molbev/msab186

Dobro, M. J., Oikonomou, C. M., Piper, A., Cohen, J., Guo, K., Jensen, T., et al. (2017). Uncharacterized bacterial structures revealed by electron cryotomography. J. Bacteriol. 199:e00100-17. doi: 10.1128/JB.00100-17

Fuerst, J. A., and Sagulenko, E. (2011). Beyond the bacterium: planctomycetes challenge our concepts of microbial structure and function. Nat. Rev. Microbiol. 9, 403-413. doi: 10.1038/nrmicro2578 development involves an asymmetric cell division followed by one cell engulfing the other. This invites parallels with the recent discovery of phagocytosis by the planctomycete, Candidatus Uab amorphum (Shiratori et al., 2019). Second, work on the giant firmicute Epulopiscium shows that its endospore-like intracellular daughter cells are formed autogenously (Angert and Clements, 2004). This is intriguing given the likely autogenous origins of several organelles, including the eukaryote nucleus. Understanding these separate origins is likely to shed light on the broader process of autogenesis (Hendrickson and Poole, 2018).

Finally, Caetano-Anollés presents an analysis of Gene Ontology terms associated with proteomes and functionomes from the three domains of life. Perhaps in contrast to the connections being made between Asgard lineages and eukaryote cell complexity, his analysis suggests that Archaea possess a small and relatively homogeneous "vocabulary" in comparison with the greater heterogeneity and complexity observed for Bacteria. Moreover, fold family analysis supports other recent suggestions of an ancient evolutionary link between Bacteria and Eukarya (Devos, 2021), perhaps consistent with some shared basal elements needed for the evolution of compartmentation.

We end with a final thought. To understand organellar evolution, it is worth considering what an organelle is. Organelles are often equated with a membrane-bounded compartment, but some of the examples highlighted here do not fit that narrow definition. It is noteworthy that the most conspicuous eukaryotic organelle, the nucleus, is continuous with the cytoplasm and dynamic, being assembled and disassembled during open mitosis. Considering other structures, such as protein-based compartmentation and spatial organization (such as in Tuwongella and the nucleolus), will be essential to investigating the origins of complex compartmentation.

\section{AUTHOR CONTRIBUTIONS}

All authors listed have made a substantial, direct and intellectual contribution to the work, and approved it for publication.

\section{FUNDING}

AP acknowledges funding support from the Royal Society Te Apārangi (18-MAU-009), New Zealand.
Gill, S., Catchpole, R., and Forterre, P. (2019). Extracellular membrane vesicles in the three domains of life and beyond. FEMS Microbiol. Rev. 43, 273-303. doi: 10.1093/femsre/fuy042

Grant, C. R., Wan, J., and Komeili, A. (2018). Organelle formation in bacteria and archaea. Annu. Rev. Cell Dev. Biol. 34, 217-238. doi: 10.1146/annurev-cellbio-100616-060908

Güttinger, S., Laurell, E., and Kutay, U. (2009). Orchestrating nuclear envelope disassembly and reassembly during mitosis. Nat. Rev. Mol. Cell Biol. 10, 178-191. doi: 10.1038/nrm2641

Hendrickson, H. L., and Poole, A. M. (2018). Manifold routes to a nucleus. Front. Microbiol. 9:2604. doi: 10.3389/fmicb.2018.02604

Imachi, H., Nobu, M. K., Nakahara, N., Morono, Y., Ogawara, M., Takaki, Y., et al. (2020). Isolation of an archaeon at the prokaryote-eukaryote interface. Nature 577, 519-525. doi: 10.1038/s41586-019-1916-6 
Katayama, T., Nobu, M. K., Kusada, H., Meng, X. Y., Hosogi, N., Uematsu, K., et al. (2020). Isolation of a member of the candidate phylum 'Atribacteria' reveals a unique cell membrane structure. Nat. Commun. 11:6381. doi: 10.1038/s41467-020-20149-5

Neumann, S., Wessels, H. J., Rijpstra, W. I., Sinninghe Damsté J. S., Kartal, B., Jetten, M. S., et al. (2014). Isolation and characterization of a prokaryotic cell organelle from the anammox bacterium Kuenenia stuttgartiensis. Mol. Microbiol. 94, 794-802, doi: 10.1111/mmi.12816

Sagulenko, E., Morgan, G. P., Webb, R. I., Yee, B., Lee, K. C., Fuerst, J. A., et al. (2014). Structural studies of planctomycete Gemmata obscuriglobus support cell compartmentalisation in a bacterium. PLoS ONE 9:e91344. doi: 10.1371/journal.pone. 0091344

Sagulenko, E., Nouwens, A., Webb, R. J., Green, K., Yee, B., Leis, A., et al. (2017). Nuclear pore-like structures in a compartmentalized bacterium. PLoS ONE 12:e0169432. doi: 10.1371/journal.pone.0169432

Santarella-Mellwig, R., Pruggnaller, S., Roos, N., Mattaj, I. W., and Devos, D. P. (2013). Three-dimensional reconstruction of bacteria with a complex endomembrane system. PLoS Biol. 11:e1001565. doi: 10.1371/journal.pbio.1001565

Shiratori, T., Suzuki, S., Kakizawa, Y., and Ishida, K.-I. (2019). Phagocytosislike cell engulfment by a planctomycete bacterium. Nat. Commun. 10:5529. doi: $10.1038 /$ s41467-019-13499-2

van Teeseling, M. C. F., and Jogler, C. (2021). Cultivation of elusive microbes unearthed exciting biology. Nat. Commun. 12:75. doi: 10.1038/s41467-020-20393-9

Wiegand, S., Jogler, M., Boedeker, C., Pinto, D., Vollmers, J., Rivas-Marín, E., et al. (2020). Cultivation and functional characterization of 79 planctomycetes uncovers their unique biology. Nat. Microbiol. 5, 126-140. doi: 10.1038/s41564-019-0 588-1

Wiegand, S., Jogler, M., and Jogler, C. (2018). On the maverick planctomycetes. FEMS Microbiol. Rev. 42, 739-760. doi: 10.1093/femsre/f uy029

Zaremba-Niedzwiedzka, K., Caceres, E. F., Saw, J. H., Bäckström, D., Juzokaite, L., Vancaester, E., et al. (2017). Asgard archaea illuminate the origin of eukaryotic cellular complexity. Nature 541, 353-358. doi: 10.1038/nature21031

Conflict of Interest: The authors declare that the research was conducted in the absence of any commercial or financial relationships that could be construed as a potential conflict of interest.

Publisher's Note: All claims expressed in this article are solely those of the authors and do not necessarily represent those of their affiliated organizations, or those of the publisher, the editors and the reviewers. Any product that may be evaluated in this article, or claim that may be made by its manufacturer, is not guaranteed or endorsed by the publisher.

Copyright $\odot 2021$ Franke, Fuerst and Poole. This is an open-access article distributed under the terms of the Creative Commons Attribution License (CC BY). The use, distribution or reproduction in other forums is permitted, provided the original author(s) and the copyright owner(s) are credited and that the original publication in this journal is cited, in accordance with accepted academic practice. No use, distribution or reproduction is permitted which does not comply with these terms. 